\title{
Enucleación transuretral con energía bipolar en el tratamiento de la hiperplasia prostática obstructiva
}

\author{
Bipolar transurethral enucleation in the treatment of \\ obstructive prostatic hyperplasia
}

\author{
Iván Azael Martínez-Alonso, Carlos Emmanuel Estrada-Carrasco, Jesús Javier Torres-Gómez, Gustavo Armando \\ Hernández-Palacios, José de Jesús Oswaldo Islas-García, Rafael Alberto Valdez-Flores, San Juan Padrón-Lucio, \\ Carlos Omar Germán-Garrido, Janette Gorostieta-Luján, José Gadú Campos-Salcedo
}

\begin{abstract}
Resumen
ANTECEDENTES: La enucleación prostática con energía bipolar puede ser una alternativa efectiva a la técnica de enucleación con láser de holmio en pacientes con adenomas de gran tamaño.

OBJETIVO: Evaluar la eficacia y seguridad de la enucleación prostática con energía bipolar en pacientes con hiperplasia prostática obstructiva.

MATERIALES Y MÉTODOS: Estudio retrospectivo, observacional, analítico y descriptivo efectuado en pacientes con indicación relativa y absoluta de tratamiento endourológico para hiperplasia prostática. Se analizaron parámetros pre y posoperatorios (seis meses) como: IPSS, calidad de vida (QoL), volumen prostático, concentración de antígeno prostático específico (APE), volumen vesical pre y posmiccional, y flujo urinario máximo (Qmax). Características perioperatorias: tiempo quirúrgico, tejido prostático enucleado, tejido enucleado por unidad de tiempo ( $\mathrm{g} / \mathrm{min}$ ), tiempo de hospitalización y de cateterización. Para el análisis estadístico se utilizó el programa Excel y SPSS.

RESULTADOS: Se registraron 30 pacientes. La indicación más común de cirugía fue retención aguda de orina (56\%) y resistencia al tratamiento farmacológico $(40 \%)$. La media del IPSS fue $22.72 \pm 7.65$ y volumen prostático $92.97 \pm 68.61$; concentración de antígeno prostático específico $6.4 \pm 4.38 \mathrm{ng} / \mathrm{mL}$, tiempo quirúrgico $113.97 \pm 52.1$ min y tejido prostático enucleado $65 \pm 56.5 \mathrm{~g}$. La eficacia de la enucleación-resección fue de $0.59 \pm 0.35 \mathrm{~g} / \mathrm{min}$, tiempo promedio de hospitalización 4.3 días y de cateterización posoperatoria 3.4 días. A seis meses de seguimiento no se reportó retención aguda de orina; la media de antígeno prostático fue $1.6 \pm 0.7$ e IPSS $4.8 \pm 2.1$. Solo dos pacientes tuvieron incontinencia urinaria de esfuerzo leve.

CONCLUSIÓN: La enucleación prostática con energía bipolar es una técnica alternativa efectiva y segura en pacientes con hiperplasia prostática obstructiva.

PALABRAS CLAVE: Enucleación prostática; enucleación con energía bipolar; hiperplasia prostática benigna.
\end{abstract}

Abstract

BACKGROUND: Bipolar enucleation of the prostate can be an effective alternative to the technique of Holmium laser enucleation in patients with large adenomas.

OBJECTIVE: To evaluate the efficacy and safety of bipolar enucleation of the prostate for the treatment of obstructive prostatic hyperplasia.

MATERIALS AND METHODS: A retrospective, observational, analytic, and descriptive study was conducted on patients indicated for relative and absolute endourologic treatment of prostatic hyperplasia. The preoperative and postoperative (six months) parameters of IPSS, quality of life (QoL), prostate volume, prostate-specific antigen
Servicio de Urología, Hospital Central Militar, Ciudad de México.

Recibido: septiembre 2018

Aceptado: septiembre 2018

Correspondencia Iván Azael Martínez Alonso navileaza@hotmail.com Twitter: @leazanavi

Este artículo debe citarse como Martínez-Alonso IA, Estrada-Carrasco $\mathrm{CE}$, Torres-Gómez JJ; Hernández-Palacios GA, Islas-García JJO, Valdez-Flores RA, Padrón-Lucio SJ, Germán-Garrido CO, Gorostieta-Luján J, Campos-Salcedo JG. Enucleación transuretral con energía bipolar en el tratamiento de la hiperplasia prostática obstructiva. Rev Mex Urol. 2018 sept-oct;78(5):366-374.

Doi: https://doi.org/10.24245/revmexurol.v78i5.2356 
(PSA) levels, pre-micturition and post-micturition bladder volumes, and maximum urinary flow rate (Qmax) were analyzed. Perioperative characteristics: surgery duration, enucleated prostate tissue; enucleated tissue per time unit ( $/ \mathrm{min})$, hospital stay, and catheterization duration. The statistical analysis was carried out using the Excel program and the SPSS statistical package.

RESULTS: Thirty patients were included in the study. Acute urinary retention was the most common indication for surgery $(56 \%)$, followed by refractoriness to medical treatment $(40 \%)$. In means, IPSS was $22.72 \pm 7.65$, prostate volume $92.97 \pm 68.61$, prostate-specific antigen $6.4 \pm 4.38 \mathrm{ng} / \mathrm{mL}$, surgery duration $113.97 \pm 52.1 \mathrm{~min}$, enucleated prostate tissue $65 \pm 56.54 \mathrm{~g}$, enucleation/resection efficacy $0.59 \pm 0.35 \mathrm{~g} /$ min, hospital stay 4.31 days, and postoperative catheterization duration 3.4 days. At month six of follow-up, none of the patients presented with acute urinary retention, mean prostate-specific antigen was $1.61 \pm 0.7$, and mean IPSS was $4.8 \pm 2.1$. Only two patients presented with mild stress urinary incontinence.

CONCLUSIONS: Bipolar enucleation of the prostate is a safe and effective alternative technique in patients with obstructive prostatic hyperplasia.

KEYWORDS: Enucleation of the prostate; Bipolar enucleation; Benign prostatic hyperplasia.

\section{ANTECEDENTES}

La obstrucción del tracto urinario inferior, secundaria a hiperplasia prostática benigna, provoca síntomas que repercuten en la calidad de vida y el estado funcional y emocional de los pacientes, incluso puede ser inevitable en sujetos de la tercera edad; por lo tanto, la enfermedad justifica el tratamiento. ${ }^{1}$

La prevalencia de síntomas de la vía urinaria inferior incrementa significativamente con la edad: $13-14 \%$ en la quinta década de la vida y $28-43 \%$ después de los 60 años. La resección transuretral de la próstata se considera el tratamiento de elección en pacientes con hiperplasia prostática obstructiva, con resultados satisfactorios según las escalas IPSS (Índice de Severidad de Síntomas Prostáticos), calidad de vida (QoL) y flujo urinario máximo (Qmax). ${ }^{2}$

Desde 1998 se practica la enucleación prostática con láser de holmio (HoLEP), con resultados equivalentes a la prostatectomía simple abierta, incluso con mayor efectividad que la resección transuretral de próstata en cuanto al alivio de los síntomas, mejor calidad de vida y parámetros urodinámicos. Las características importantes de la HoLEP incluyen: tratamiento de próstata de gran volumen, seguridad durante el procedimiento y mejoría por periodo prolongado; sin embargo, entre sus desventajas se encuentra la elevada curva de aprendizaje y el alto costo de la técnica. ${ }^{3}$

Las técnicas de enucleación con otros tipos de energía han surgido como alternativa a la HoLEP, pues la enucleación completa del adenoma se asocia con mejoría por largos periodos, lo que repercute de manera notable en los índices: QoL, Qmax e IPSS. Con el advenimiento de las técnicas de enucleación, la tasa de reintervención ha disminuido a $1.4 \% .^{4}$

La técnica de enucleación prostática con energía bipolar es una adaptación a la técnica convencional con láser, lo que representa un cambio en el paradigma del tratamiento de la hiperplasia prostática. 
El objetivo de este estudio fue: evaluar la eficacia y seguridad de la enucleación prostática con energía bipolar en pacientes con hiperplasia prostática obstructiva.

\section{MATERIALES Y MÉTODOS}

Estudio retrospectivo, observacional, analítico y descriptivo, efectuado en el servicio de Urología del Hospital Central Militar de la Ciudad de México, entre los años 2015-2017. Se seleccionaron pacientes operados de enucleación prostática con energía bipolar, mayores de 50 años, con síntomas de la vía urinaria inferior secundarios a hiperplasia prostática obstructiva, tacto rectal no sospechoso de la enfermedad e indicación de cirugía endourológica por obstrucción del orificio de salida de la vejiga (hematuria de origen prostático, infección recurrente de vías urinarias, litiasis vesical, retención aguda de orina, síntomas obstructivos urinarios crónicos resistentes al tratamiento farmacológico y enfermedad renal de origen posrenal) y estimación del volumen prostático mediante ultrasonido transrectal. Criterios de exclusión: pacientes con discrasias sanguíneas, síntomas relacionados con vejiga neurogénica, obstrucción infravesical diferente a la prostática (estenosis uretral), hipo o epispadias, sujetos con antecedente de tratamiento endourológico de próstata, infección de vías urinarias no tratada, urocultivo positivo sin impregnación con antibióticos selectivos por antibiograma, diagnóstico de cáncer de próstata por concentración elevada de antígeno prostático específico o cualquier hallazgo sugerente de tumores uroteliales en la cistoscopia inicial y quienes no aceptaran el procedimiento.

Se utilizó un resectoscopio Storz ${ }^{\circledR} 26$ Fr con asa Supersect, parámetros de $120 \mathrm{~W}$ de corte y $80 \mathrm{~W}$ de coagulación y solución fisiológica de $\mathrm{NaCl}$ 0.9\% para irrigación vesical. El procedimiento quirúrgico lo efectuó un solo cirujano. La técnica inicial consistió en cistoscopia, con la finalidad de evaluar la coaptación de los lóbulos prostáticos y la coexistencia del lóbulo medio, verificando la distancia de los orificios ureterales al cuello vesical. La descripción de la técnica fue: demarcación del verum montanum, incisión con corte a las 5 y 7 de las manecillas del reloj hasta la cápsula. Se realizó un corte después del verum montanum hasta la porción de la cápsula y disección roma del adenoma de manera retrógrada, combinando de forma mecánica el uso del asa bipolar y del resectoscopio; en los sitios de sangrado de los vasos prostáticos se realizó coagulación y ante la observación de adherencias del adenoma a la cápsula se efectuó el corte localizado. El parámetro de referencia de la disección fue el espacio virtual entre el adenoma y la cápsula, y como referencia lateral el corte realizado previamente. Después de la disección en el cuello de la vejiga se dejó de manera intencionada el adenoma fijo al cuello, con la intención de realizar, como procedimiento final, la resección mediante la técnica de hongo y evitar el uso de morcelador. Para la enucleación de los lóbulos laterales se prolongó el corte del radio de las 7 de manera lateral, adyacente al verum montanum, hasta el borde, donde se visualizó el inicio del adenoma, corte que se extendió hasta la cápsula y se prolongó de manera circunferencial hasta el radio de las 11 y 1 , con la finalidad de evitar dañar mecánica y térmicamente el esfínter urinario. De manera anterógrada se enucleó el adenoma combinando la disección del asa y del resectoscopio mecánicamente, mediante el corte localizado en las adherencias adenoma-capsulares y coagulación en los sitios de sangrado, según correspondiera. La disección en ambos lóbulos laterales se prolongó de manera retrógrada en toda la circunferencia del adenoma hasta el radio de las 11 y 1 , dejando, intencionadamente, fijos por una base dependiente de la parte superior de la próstata, para resecar con la técnica de hongo. Los fragmentos fueron retirados con evacuador tipo granada y se colocó una sonda de 3 vías tipo 
Rusch, con irrigación continua que se suspendió a criterio del cirujano.

Se analizaron parámetros preoperatorios como: IPSS, calidad de vida (QoL), volumen prostático, concentración de antígeno prostático específico (APE), volumen vesical pre y posmiccional, y flujo urinario máximo (Qmax). Características perioperatorias: tiempo quirúrgico, tejido prostático enucleado, tejido enucleado por unidad de tiempo (g/min), tiempo de hospitalización y de cateterización. Después de seis meses del posoperatorio se analizaron los resultados mediante las escalas IPSS, QoL, volumen residual posmiccional, Qmax y determinación de la concentración de antígeno prostático. Para el análisis estadístico se utilizó el programa Excel y SPSS.

\section{RESULTADOS}

De acuerdo con la revisión de los archivos médicos se registraron 30 pacientes. El Cuadro 1 muestra las características preoperatorias de los casos analizados. La indicación más común de cirugía fue retención aguda de orina (56\%), seguida de resistencia al tratamiento farmacológico (40\%). El promedio de tiempo quirúrgico

Cuadro 1. Características preoperatorias $(n=30)$

\begin{tabular}{lcc}
\hline Parámetro & Media \pm DE & Rango \\
\hline Edad & $70.52 \pm 8.23$ & $92-55$ \\
\hline IPSS & $22.72 \pm 7.65$ & $35-13$ \\
\hline QoL & $4.76 \pm 0.44$ & $5-4$ \\
\hline Volumen prostático $(\mathrm{mL})$ & $92.97 \pm 68.61$ & $400-30$ \\
\hline APE $(\mathrm{ng} / \mathrm{mL})$ & $6.4 \pm 4.38$ & $18.5-1.12$ \\
\hline $\begin{array}{l}\text { Volumen premiccional } \\
(\mathrm{mL})\end{array}$ & $415.52 \pm 135.14$ & $500-64$ \\
\hline $\begin{array}{l}\text { Volumen posmiccional } \\
(\mathrm{mL})\end{array}$ & $346.52 \pm 193.49$ & $500-16$ \\
\hline $\begin{array}{l}\text { Qmax }(\mathrm{mL} / \mathrm{min}) \\
\end{array}$ & $2.9 \pm 3.86$ & $12-0$
\end{tabular}

IPSS: escala de severidad de síntomas prostáticos; QoL: cuestionario de calidad de vida; APE: antígeno prostático específico; Qmax: flujo urinario máximo. fue de $113.97 \pm 52.1$ minutos; tejido prostático enucleado $65 \pm 56.5 \mathrm{~g}$. El análisis de eficacia de la enucleación-resección de tejido prostático por unidad de tiempo resultó en $0.59 \pm 0.35 \mathrm{~g} /$ min; tiempo de hospitalización $4.3 \pm 5$ días y de cateterización $3.4 \pm 2.1$ días (Cuadro 2). Ningún paciente ameritó transfusión de hemoderivados; solo se registró un caso con estancia hospitalaria mayor de 30 días debida a un diagnóstico diferente al urológico y otro contaba con urocultivo positivo previo a la cirugía, a quien se indicó la aplicación selectiva de antibióticos de acuerdo con el antibiograma. El análisis y seguimiento a seis meses del posoperatorio no reportó pacientes con cáncer de próstata, según el tejido de patología. Respecto de los parámetros preoperatorios, se encontró diferencia estadísticamente significativa en: disminución del puntaje en IPSS $(4.8 \pm 2.1, p<0.001)$, concentración de antígeno prostático $(1.6 \pm 0.78 \mathrm{ng} / \mathrm{mL}, \mathrm{p}<0.001)$, volumen de orina residual posmiccional $(18.4 \pm 13.9$ $\mathrm{mL}, \mathrm{p}<0.001$ ), mejoría en el cuestionario de calidad de vida ([QoL] $1.38 \pm 0.78, p<0.001)$ y en los parámetros urodinámicos ([Qmax] $20 \pm 5.4$, $\mathrm{p}<0.001)$ Cuadro 3. No se reportaron pacientes con complicaciones posoperatorias inmediatas que ameritaran transfusiones o prolongaran su estancia hospitalaria. Un paciente permaneció hospitalizado por más de 30 días (tratamiento por un cuadro depresivo crónico) y dos manifestaron incontinencia urinaria de esfuerzo, cuyo tratamiento consistió en duloxetina y terapia

Cuadro 2. Características perioperatorias

\begin{tabular}{l|c|c|}
\hline Parámetro & Media \pm DE & Rango \\
\hline $\begin{array}{l}\text { Tiempo quirúrgico (min) } \\
\text { Tejido prostático enucleado } \\
\text { (g) }\end{array}$ & $65 \pm 56.54$ & $(320-20)$ \\
\hline $\begin{array}{l}\text { Tejido enucleado-resecado } \\
\text { por unidad de tiempo (g/min) }\end{array}$ & $0.59 \pm 0.35$ & $(1.5-0.2)$ \\
$\begin{array}{l}\text { Tiempo de hospitalización } \\
\text { (días) }\end{array}$ & $4.31 \pm 5.03$ & $(30-3)$ \\
$\begin{array}{l}\text { Tiempo de cateterización } \\
\text { (días) }\end{array}$ & $3.45 \pm 2.13$ & (7-1)
\end{tabular}


del piso pélvico, con lo que mostraron mejoría en la calidad de vida (requirieron el uso de 1-2 pañales al día, por goteo con el esfuerzo físico).

Cuadro 3. Comparación entre parámetros preoperatorios y posoperatorios (seis meses)

\begin{tabular}{|c|c|c|c|}
\hline Parámetro & Preoperatorio & $\begin{array}{c}6 \text { meses del } \\
\text { posoperatorio }\end{array}$ & $\mathbf{p}$ \\
\hline IPSS & $22.72 \pm 7.65$ & $4.83 \pm 2.14$ & $\begin{array}{c}< \\
0.001\end{array}$ \\
\hline $\begin{array}{l}\text { QoL } \\
(\mathrm{mL} / \mathrm{min})\end{array}$ & $4.76 \pm 0.44$ & $1.38 \pm 0.78$ & $\begin{array}{c}< \\
0.001\end{array}$ \\
\hline $\begin{array}{l}\text { Volumen } \\
\text { posmiccional } \\
(\mathrm{mL})\end{array}$ & $\begin{array}{c}346.52 \\
\pm 193.49\end{array}$ & $18.46 \pm 13.93$ & $\begin{array}{c}< \\
0.001\end{array}$ \\
\hline Qmax & $2.9 \pm 3.86$ & $20.04 \pm 5.44$ & $\begin{array}{c}< \\
0.001\end{array}$ \\
\hline APE (ng/mL) & $6.4 \pm 4.38$ & $1.61 \pm 0.78$ & $\begin{array}{c}< \\
0.001\end{array}$ \\
\hline
\end{tabular}

IPSS: escala de severidad de síntomas prostáticos; QoL: cuestionario de calidad de vida; APE: antígeno prostático específico; Qmax: índice del flujo urinario máximo.

\section{DISCUSIÓN}

En la actualidad el urólogo cuenta con diferentes opciones de tratamiento quirúrgico para pacientes con hiperplasia prostática obstructiva, de los que sobresale la enucleación prostática con láser de holmio como el patrón de referencia; sin embargo, no se encuentra disponible en la mayor parte de los centros hospitalarios, por las características propias de la técnica, los recursos económicos para solventar la tecnología y la curva de aprendizaje demasiado alta. ${ }^{5}$ De esta forma, la enucleación con energía bipolar se ha desarrollado como opción adicional a la enucleación con láser de holmio. En México existen pocas instituciones que cuentan con esta técnica, incluso son escasos los reportes de la bibliografía al respecto.

En este estudio se observó que la enucleación con energía bipolar disminuyó los síntomas y parámetros funcionales entre el primero y sexto mes de seguimiento, evidenciándose mejoría en el puntaje del IPSS $(22.7 \pm 7.6$ vs $4.8 \pm 2.1)$, Qmax $(2.9 \pm 3.8$ vs $20 \pm 5.4 \mathrm{~mL} / \mathrm{min})$ y volumen de orina residual $(346.5 \pm 193.5$ a $18.5 \pm$ $13.9 \mathrm{~mL}$ ), este último considerado un parámetro importante en la evaluación posquirúrgica. Diversos estudios han evaluado estos parámetros en relación con la técnica de enucleación prostática con láser de holmio; por ejemplo, Elzayat y sus colegas reportaron mejoría en el Qmax (6.3 a $16.2 \mathrm{~mL} / \mathrm{seg}$ ) e IPSS (17.3 a 5.6 puntos). ${ }^{6}$ Un punto importante en nuestro estudio, que no lo hace equiparable con los demás, es que en la mayor parte de las investigaciones los pacientes egresan el mismo día de la cirugía; sin embargo, en nuestra institución, según los protocolos de atención médica, todos los pacientes intervenidos quirúrgicamente se dan de alta al siguiente día de la cirugía, por lo que el promedio de hospitalización fue de 4.1 días, lo que supuso el incremento de estancia hospitalaria.

Las técnicas de enucleación proporcionan mayor extracción de adenoma prostático, lo que sugiere mejoría notable en los resultados. El tamaño promedio de las próstatas extirpadas fue de $92.8 \pm 68.6$ cc, logrando una extracción de tejido promedio de $65 \pm 56.5 \mathrm{~g}$, con amplio rango de volúmenes prostáticos, por lo que en la actualidad se aplica un nuevo concepto que evalúa la eficacia de la enucleación-resección por unidad de tiempo. La investigación de Kawamura y su grupo, en la que utilizaron la técnica de enucleacion con energía bipolar, reportó una eficacia de $0.32 \mathrm{~g} / \mathrm{min}(0.18-0.55 \mathrm{~g} / \mathrm{min})$ de tejido enucleado. 7 Por su parte, Hirasawa y sus coautores reportaron la mayor casuística de casos tratados con esta técnica y encontraron eficacia de $0.54 \pm 0.01 \mathrm{~g} / \mathrm{min} .{ }^{8}$ En relación con esta variable, la eficacia de la técnica en nuestro estudio fue de $0.59 \pm 0.35 \mathrm{~g} / \mathrm{min}$. Los resultados de las series mencionadas coinciden con los obtenidos mediante enucleación láser, con 
eficacia promedio de $0.52 \mathrm{~g} / \mathrm{min}^{9}$. La eficacia de la técnica puede deberse a que la resección de un adenoma isquémico y sin sangrado mejora la confianza del urólogo al momento de la extirpación, lo que aumenta la velocidad y profundidad del corte, maniobras que se traducen en eficiencia de tiempo.

Por cuestiones relacionadas con la técnica de enucleación con energía bipolar, el tiempo de resección es más prolongado, principalmente en la disección cuidadosa y endoscópica del adenoma de la cápsula y, además, la extracción del tejido por morcelación o resección; sin embargo, comparado con otras técnicas, el tiempo en que se realiza el procedimiento es similar. Feng y sus colaboradores compararon las características perioperatorias entre la enucleación con láser de Thulio y la enucleación con energía bipolar, pero no encontraron diferencia significativa en los tiempos de resección $(67.9 \pm 20.8$ vs 69.21 $\pm 19.8 \mathrm{~min}$ ). En nuestro ensayo, el tiempo total de cirugía fue de $113.9 \pm 52.1$, quizá porque la media de tejido prostático enucleado fue mayor (65 \pm 56.54 g, límites 320-20 cc) y por la experiencia inicial de la resección mediante esta técnica. ${ }^{10}$

La enucleación prostática es una técnica difícil de dominar para el urólogo, pues requiere una curva de aprendizaje alta. De acuerdo con diversos estudios, un urólogo sin adiestramiento puede obtener una experiencia razonable después de 50 casos, con resultados comparables a los expertos. ${ }^{9,11}$ Respecto de la enucleación con energía bipolar, la serie más grande analizó que la curva de aprendizaje disminuye marcadamente después de los 50 procedimientos, al igual que para la enucleación con láser. En nuestro estudio, todos los procedimientos fueron practicados por un urólogo con experiencia previa en enucleación prostática con láser de holmio, lo que puede explicar los tiempos similares a los reportados en la bibliografía. ${ }^{8,12}$
Un dato importante es la notable disminución de la concentración de antígeno prostático específico (APE) después de la enucleación, lo que sugiere una eficacia importante de la técnica. La mayor parte de los estudios indica disminución del APE (80.4\%) en pacientes intervenidos con enucleación con láser de holmio (HoLEP) de $80.4 \%,{ }^{13}$ comparado con estudios que realizan resección transuretral de la próstata $(70 \%) .{ }^{14} \mathrm{La}$ serie actual más grande de casos de enucleación con energía bipolar reporta una mejoría de $80.2 \% .{ }^{8}$ En nuestro estudio, la concentración de APE disminuyó $75 \%$, cifra comparable con la técnica láser de holmio; sin embargo, es importante mencionar que la mayoría de los pacientes tenía concentraciones elevadas de APE y cáncer de próstata previamente descartado. Un porcentaje pequeño de pacientes reporta cáncer de próstata de forma fortuita (4.8-7.6\%). En nuestro estudio, por considerar un tamaño reducido de muestra y descartar mediante biopsia el diagnóstico de cáncer de próstata, no se establecieron diagnósticos fortuitos según el estudio de patología. ${ }^{15}$

El Cuadro 4 expone los resultados asociados con la técnica de enucleación con energía bipolar en diferentes series de todo el mundo. Aunque la técnica de extracción del adenoma varía de una institución a otra, los resultados son similares en la mayor parte de los centros que utilizan esta técnica. ${ }^{16,17}$

Las complicaciones asociadas con las técnicas de enucleación incluyen: retención urinaria, sangrado posoperatorio inmediato, infección e incontinencia urinaria. ${ }^{18}$ En este estudio no registramos pacientes con sangrado que ameritaran transfusiones o reintervención quirúrgica; no obstante, 3/30 casos requirieron el retiro de la sonda a los siete días posquirúrgicos debido a retención urinaria y 2/30 tuvieron complicaciones tardías, específicamente incontinencia urinaria de esfuerzo leve (goteo), cuyo tratamiento consistió 


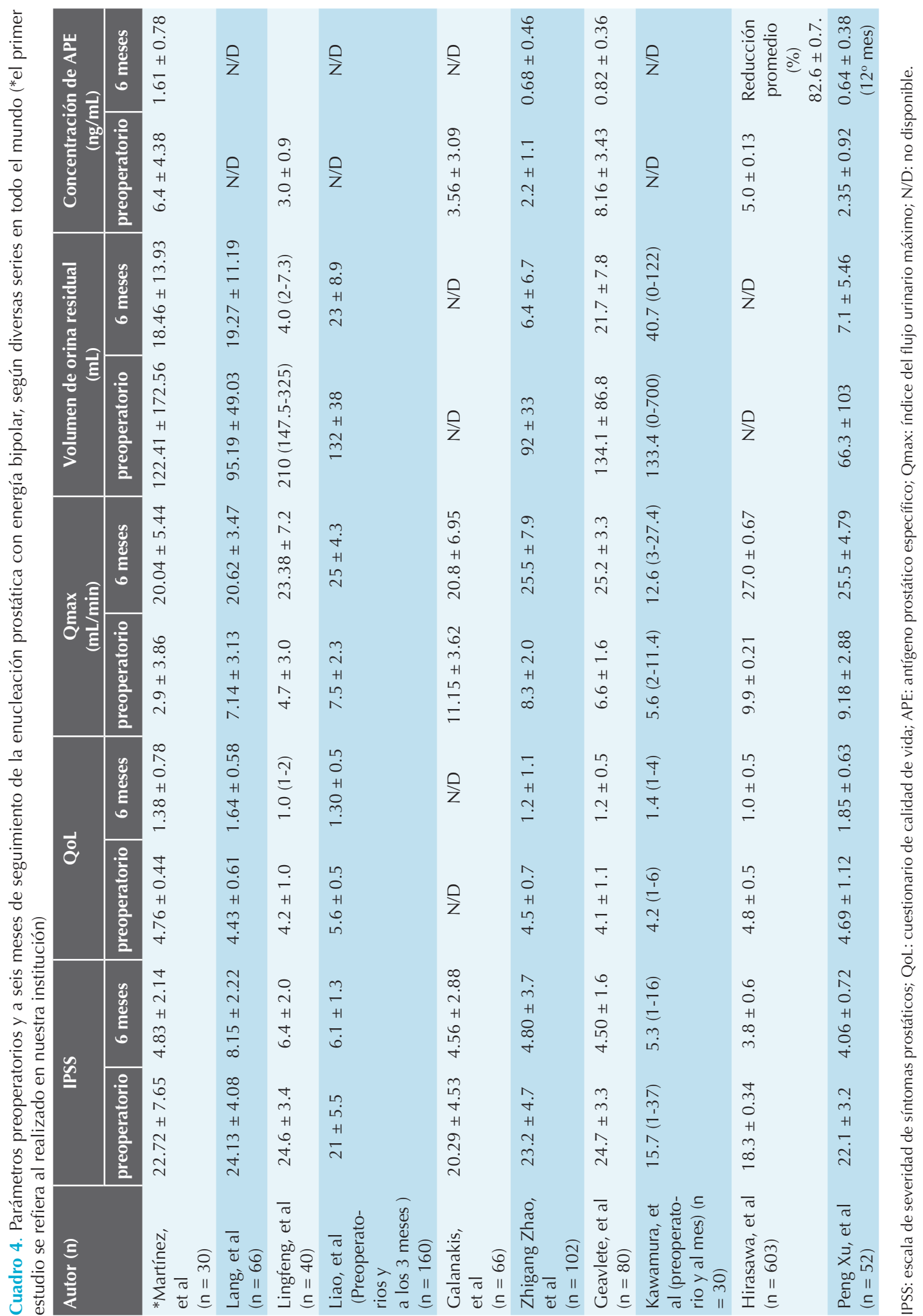


en $\alpha$-agonistas y uso de 1-2 pañales al día. La incontinencia urinaria provocada por las técnicas de enucleación se ha reportado en $20 \%$ de los casos, principalmente en sujetos intervenidos con láser de holmio. En la serie más grande de enucleación con energía bipolar se registró una tasa de incontinencia a seis meses de seguimiento de $3.3 \%$, con curación completa al finalizar el año posquirúrgico. De acuerdo con estos datos, la cronicidad de los síntomas, el tamaño de la próstata y la edad del paciente representan factores importantes de complicaciones posquirúrgicas. ${ }^{19,20,21}$

Aunque exista una mínima manipulación del esfínter durante los procedimientos endourológicos, la tracción retrógrada supone el principal factor de incontinencia urinaria. Walz y sus colegas reportaron que el esfínter urinario, por tratarse de una capa interna de músculo liso, tiene mayor posibilidad de dañarse por la tracción mecánica de las fibras longitudinales internas alrededor del ápex de la glándula, originando un daño transitorio, factores independientes a la técnica como: cronicidad de los síntomas, cirugías previas, edad del paciente y volumen de la próstata, que representan factores no estudiados y pueden tener relevancia como consecuencia de la incontinencia urinaria después del procedimiento quirúrgico. ${ }^{8,22}$ De esta forma, antes de efectuar cualquier técnica de enucleación se requiere el conocimiento de la anatomía endourológica de la próstata, con la finalidad de evitar un daño colateral y llevar a cabo un procedimientos efectivo.

En la Figura 1 tabulamos el costo de hospitalización del total de pacientes de este estudio, a 4.3 días de estancia hospitalaria (tiempo medio considerado en este ensayo). Compara el costos de hospitalización y el que conlleva utilizar los diferentes tipos de energía disponibles para el tratamiento de la hiperplasia prostática benigna como: energía bipolar (enucleación bipolar), HoLEP y láser verde (Green Light). Las barras muestran la variación del costo total de

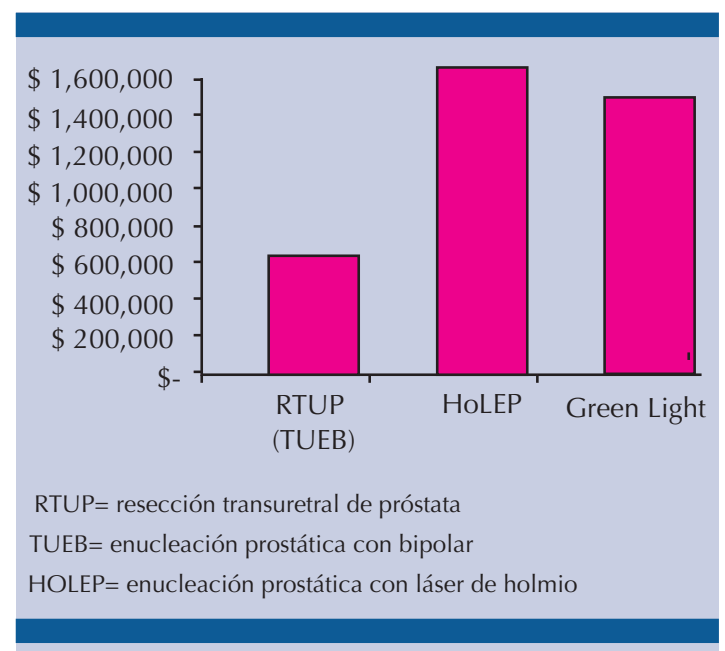

Figura 1. Análisis de costos según las técnicas de enucleación prostática.

hospitalización relacionada con el uso de los diferentes tipos de energía, donde el costo más bajo lo ofrece la energía bipolar. Este esquema es únicamente demostrativo, por lo que es importante mencionar la factibilidad que pudiera tener esta técnica en instituciones de salud que no cuenten con los medios económicos para indicar métodos más modernas y se desee poner al servicio de los pacientes técnicas de enucleación. Es importante mencionar que los costos entre las instituciones varían de acuerdo con sus protocolos y licitaciones; sin embargo, se recalca la notable diminución del costo hospitalario al ofrecer este tipo de energía.

Las limitaciones de este estudio incluyen su tipo retrospectivo, que el procedimiento quirúrgico lo haya efectuado un solo cirujano y la cantidad de pacientes incluidos en la investigación. Se requieren estudios adicionales que evalúen la función sexual y complementen a largo plazo la evaluación urodinámica, con la finalidad de ampliar el seguimiento de los pacientes, como lo sugieren diversas investigaciones asociadas con enucleación bipolar. También es necesario efectuar estudios multicéntricos en México para determinar la viabilidad de esta técnica. 


\section{CONCLUSIÓN}

La enucleación prostática con energía bipolar es un método seguro y efectivo en pacientes con hiperplasia prostática obstructiva, sobre todo en quienes tienen adenomas de gran tamaño, destaca la mejoría durante un periodo prolongado. Esta técnica de enucleación es similar a otras descritas a nivel mundial. Entre sus desventajas se encuentran el tiempo quirúrgico prolongado y la curva de aprendizaje alta. Se requieren estudios prospectivos y multicéntricos que comparen la enucleación con energía bipolar con otro tipo de técnicas para determinar su eficacia y seguridad en pacientes con hiperplasia prostática obstructiva.

\section{REFERENCIAS}

1. Mamoulakis C, et al. Bipolar versus monopolar transurethral resection of the prostate: a systematic review and meta-analysis of randomized controlled trials. Eur Urol 2009;56:798-809. doi: 10.1016/j.eururo.2009.06.037.

2. Bhojani N, et al. Morbidity and mortality after benign prostatic hyperplasia surgery: data from American College of Surgeons National Surgical Quality Improvement Program. J Endourol 2014;28:831-840. doi: 10.1089/end.2013.0805.

3. Elshal AM, et al. Green Light ${ }^{\mathrm{TM}}$ laser XPS photoselective vapo-enucleation versus holmium laser enucleation of the prostate for the treatment of symptomatic benign prostatic hyperplasia: a randomized controlled study. J Urol 2015;193:927-934. DOI: 10.1016/j.juro.2014.09.097

4. Gilling P, Williams A. Holmium laser enucleation of the prostate is the single best treatment for benign prostatic hyperplasia refractory to medication. J Endourol 2008;22:2113-2115. DOI: 10.1089/end.2008.9732

5. $\mathrm{Xu} \mathrm{A}$, et al. A randomized trial comparing diode laser enucleation of the prostate with plasmakinetic enucleation and resection of the prostate for the treatment of benign prostatic hyperplasia. J Endourol 2013; 27:1254-60. DOI: 10.1089/end.2013.0107

6. Elzayat EA, Elhilali MM. Holmium laser enucleation of the prostate (HoLEP): Long-term results, reoperation rate, and possible impact of the learning curve. Eur Urol 2007;52:1465-1471.

7. Kawamura Y, et al. clinical outcomes of transurethral enucleation with bipolar for benign prostatic hypertrophy. J Exp Clin Med 2015;40:132-136.

8. Hirasawa Y, Kato Y, Fujita K. Transurethral enucleation with bipolar for benign prostatic hyperplasia: 2-year outcomes and the learning curve of a single surgeon's experience of 603 consecutive patients. J Endourol 2017;31:679:685. DOI: 10.1089/end.2017.0092
9. Shah HN, et al. Prospective evaluation of the learning curve for holmium laser enucleation of the prostate. J Urol 2007; 177:1468-1474. DOI: 10.1016/j.juro.2006.11.091

10. Feng $L$, et al. Thulium laser enucleation versus plasmakinetic enucleation of the prostate: a randomized trial of a single center. J Endourol 2016;30:665-670. DOI: 10.1089/ end.2015.0867

11. Seki $\mathrm{N}$, et al. Holmium laser enucleation for prostatic adenoma: Analysis of learning curve over the course of 70 consecutive cases. J Urol 2003;170:1847-1850. DOI: 10.1097/01.ju.0000092035.16351.9d

12. Brunckhorst $\mathrm{O}$, et al. Evaluation of the learning curve for holmium laser enucleation of the prostate using multiple outcome measures. Urology 2015;86:824-829. DOI: 10.1016/j.urology.2015.07.021

13. Vavassori I, et al. Three-year outcome following holmium laser enucleation of the prostate com- bined with mechanical morcellation in 330 consecutive patients. Eur Urol 2008;53:599-604. DOI: 10.1016/j.eururo.2007.10.059

14. Aus $\mathrm{G}$, et al. Reference range of prostate-specific antigen after transurethral resection of the prostate. Urology 1996;47:529-531. DOI: 10.1016/S0090-4295(99)80489-0

15. Naspro R, et al. Holmium laser enucleation of the prostate versus open prostatectomy for prostates $>70 \mathrm{~g}$ : 24 -month follow-up. Eur Urol 2006;50:563-568. DOI: 10.1016/j.eururo.2006.04.003

16. Galanakis I, et al. Evolving transurethral resection of the prostate: enucleation-resection of the prostate. J Endourol 2010;24:2043-2046. DOI: 10.1089/end.2010.0300

17. Zhao Z, et al. A prospective, randomised trial comparing plasmakinetic enucleation to standard transurethral resection of the prostate for symptomatic benign prostatic hyperplasia: three-year follow-up results. Eur Urol 2010;58:752-758. DOI: 10.1016/j.eururo.2010.08.026

18. Placer J, et al. Holmium laser enucleation of prostate: Outcome and complications of self-taught learning curve. Urology 2009;73:1042-1048. DOI: 10.1016/j.urology.2008.12.052

19. Kuntz RM, et al. Does perioperative out- come of transurethral holmium laser enucleation of the prostate depend on prostate size? J Endourol 2004;18:183-188. DOI: 10.1089/089277904322959842

20. Montorsi $\mathrm{F}$, et al. Holmium laser enucleation versus transurethral resection of the prostate: Results from a 2-center, prospective, randomized trial in patients with obstructive benign hyperplasia. J Urol 2008;179(5 Suppl):S87-90. DOI: 10.1016/j.juro.2008.03.143

21. Zhu L, et al. Electrosurgical enucleation versus bipolar transurethral resection for prostates larger than $70 \mathrm{ml}$ : a prospective, randomized trial with 5-year followup. J Urol 2013;189;1427-1431. DOI: 10.1016/j.juro.2012.10.117

22. Walz J, et al. A critical analysis of the current knowledge of surgical anatomy related to optimization of cancer control and preservation of continence and erection in candidates for radical prostatectomy. Eur Urol 2010;57:179-192. DOI: 10.1016/j.eururo.2009.11.009 